\title{
Effectivness of specific mobile health applications (mHealth-apps) in gestational diabtetes mellitus: a systematic review
}

\author{
Claudia Eberle*, Maxine Loehnert and Stefanie Stichling
}

\begin{abstract}
Background: Gestational diabetes mellitus (GDM) emerges worldwide and is closely associated with short- and long-term health issues in women and their offspring, such as pregnancy and birth complications respectively comorbidities, Type 2 Diabetes (T2D), metabolic syndrome as well as cardiovascular diseases. Against this background, mobile health applications (mHealth-Apps) do open up new possibilities to improve the management of GDM. Therefore, we analyzed the clinical effectiveness of specific mHealth-Apps on clinical health-related short and long-term outcomes in mother and child.

Methods: A systematic literature search in Medline (PubMed), Cochrane Library, Embase, CINAHL and Web of Science Core Collection databases as well as Google Scholar was performed. We selected studies published 2008 to 2020 analyzing women diagnosed with GDM using specific mHealth-Apps. Controlled clinical trials (CCT) and randomized controlled trials (RCT) were included. Study quality was assessed using the Effective Public Health Practice Project (EPHPP) tool.
\end{abstract}

Results: In total, $n=6$ publications ( $n=5$ RCTs, $n=1$ CCT; and $n=4$ moderate, $n=2$ weak quality), analyzing $n=408$ GDM patients in the intervention and $n=405$ in the control groups, were included. Compared to control groups, fasting blood glucose, 2-h postprandial blood glucose, off target blood glucose measurements, delivery mode (more vaginal deliveries and fewer (emergency) caesarean sections) and patient compliance showed improving trends.

Conclusion: mHealth-Apps might improve health-related outcomes, particularly glycemic control, in the management of GDM. Further studies need to be done in more detail.

Keywords: Gestational diabetes mellitus, Medical apps, mHealth-apps, mHealth, Pregnancy, Smartphones

\section{Background}

Gestational diabetes mellitus (GDM) emerges worldwide. In 2019 and with reference to the International Diabetes Federation (IDF), approx. 20 million or approx. 16\% of live births had some form of hyperglycemia in pregnancy, approx. $84 \%$ were diagnosed with GDM [1]. Globally, the

*Correspondence: Claudia.eberle@hs-fulda.de

Medicine with specialization in Internal Medicine and General Medicine, Hochschule Fulda - University of Applied Sciences, Leipziger Strasse 123, 36037 Fulda, Germany prevalence of GDM ranged between approx. $2.1 \%$ and approx. $37.5 \%$ in 2019 [2]. Risk factors leading to GDM include maternal age, obesity, gestational weight gain $[3,4]$. GDM is associated with adverse pregnancy and birth outcomes for mother and child, eg, increased risks of preeclampsia, caesarean sections, macrosomia and shoulder dystocia $[3,5]$.

Having the concept of transgenerational programming ("fetal or perinatal programming") in mind, intra-uterine exposure to hyperglycemia "programs" the offspring to eg, obesity, glucose intolerance, T2D, insulin resistance, 
metabolic syndrome, high blood pressure, and cardiovascular diseases [6-10]. In this context, early strategies are needed to improve the management of GDM effectively. Mobile health (mHealth), especially mobile health applications (mHealth-Apps), open up innovative strategies to improve clinical outcomes in the management of GDM [11]. mHealth defines the support of medical procedures and health care measures through movile devices such as smartphones [12]. mHealth-Apps allow a more specific and individual management of patient care [13]. We recently analyzed the usage behavior of GDM related mHealth-Apps und their components [11, $14,15]$. Although smartphone apps may be beneficial in self-management, there is still a lack of individualized diabetes therapy during pregnancy $[11,13-15]$. There is evidence that smartphone-supported GDM management may improve glycemic control and compliance of GDM patients and may decrease the numbers of "off-target" measurements as well as insulin dosages and complication rates in gestational diabetics and their children [11, 16]. For example, Xie et al. [17] examined telemedical interventions in GDM patients, including mHealth apps and other (mostly web-based) applications, and found an improvement in clinical outcomes such as glycemic control. We systematically reviewed studies that evaluated the effectiveness of GDM specific mHealth-Apps on health-related outcomes in GDM patients compared to control groups.

\section{Methods}

\section{Search strategy and eligibility criteria}

We performed a systematic search in Medline (PubMed), Cochrane Library, Embase, CINAHL and Web of Science Core Collection databases based on the "Preferred Reporting Items for Systematic Reviews and Meta-Analyses (PRISMA)" guidelines [18]. The search strategy included the following keywords: "smartphone" OR "mobile phone" OR "cell phone" OR "iOS" OR "android" AND "mobile application" OR "app" AND "gestational diabetes mellitus". For example, the strategy in PubMed was as follows: (("Diabetes, Gestational”[Mesh]) AND ((((“"Smartphone”[Mesh]) OR ("Cell Phone"[Mesh])) OR ("mobile phone"[Title/ Abstract])) OR (ios [Title/Abstract])) OR (android [Title/ Abstract]))) AND ((app [Title/Abstract]) OR ("Mobile Applications"[Mesh])). We manually searched reference lists and Google Scholar to identify further studies.

We included studies that evaluated mHealth-Apps in GDM management compared to usual care (control group), and reported clinical parameters such as glycemic control, pregnancy, birth and neonatal outcomes. We involved peer-reviewed randomized controlled trials and clinical controlled trials. We have filtered the studies by year (January 2008 to November 2020) and by language (German and English). The studies were screened and selected by two independent reviewers. Moreover, we excluded poster, comments, study protocols, duplicates and studies addressing GDM diagnosis and prevention.

After removing duplicates, we scanned the titles and abstracts of the remaining studies, and reviewed the full texts.

\section{Data extraction and analysis}

We extracted author, year, design, intervention and control group, sample size, main findings related to the outcomes of interest, and significances. The results were structured by outcomes: glycemic control outcomes (1), pregnancy and birth related outcomes (2), and neonatal outcomes (3).

\section{Risk of bias assessment}

Risk of bias was assessed using the Effective Public Health Practice Project (EPHPP) tool $[19,20]$. The tool consists of the following components: selection bias, study design, confounders, blinding, data collection methods, and withdrawals [19]. EPHPP is a validated instrument to assess studies with health-related topics and appropriate for quantitative intervention studies. The tool rates the study quality as strong, moderate or weak.

\section{Results}

\section{Study characteristics}

Overall, $n=121$ records were identified through database and manual searching. After removing duplicates, we screening $n=72$ titles and abstracts and excluded $n=60$ unsuitable papers. The reasons for exclusion are documented in the PRISMA flowchart (Multimedia Appendix 1). After assessing $n=12$ papers with full-text, $n=6$ inappropriate studies were excluded. Finally, we included $\mathrm{n}=6$ eligible studies in this systematic review analyzing $n=408$ GDM patients in the intervention and $n=405$ in the control groups. These studies were divided into $n=5$ two-arm randomized controlled trials (RCT) [16, 21-24] and $n=1$ controlled clinical trial (CCT) [25]. Additionally, in the CCT, a group of $n=50$ women without GDM was included as a second control group [25]. Multimedia Appendix 2 gives an overview of included studies regarding study design, number of participants, main features of the investigated mHealt-apps, and main outcomes. We structured the outcomes in glycemic control, pregnancyand birth-related as well as neonatal outcomes.

Four of the studies reported the majority of their participants being in the third trimester of pregnancy (mean gestational age in intervention groups: $29.1( \pm 1.9)$ weeks [22], $30.9( \pm 3.6)$ weeks [24], $31.2( \pm 4.1)$ weeks [23] and $n=88(76.4 \%)$ being between 25th and 32nd gestational 
week [21]). Two studies reported only the values of gestational age used as inclusion criterion (gestational age: $<34$ weeks [16], between $14+0$ and $34+6$ [25]). Mean maternal age differed in the studies between $31.2( \pm 4.1)$ [23] and $33.9( \pm 5.5)$ years [24].

The risk of bias assessment using EPHPP tool is allocated in Multimedia Appendix 3. The quality of four studies was rated as "moderate" [16, 21, 23, 24], .whereas the quality of the two remaining studies was rated as "weak" [22, 25]. The relevant component that was crucial for low quality ratings was the lack of blinding.

\section{Glycemic control outcomes}

Multimedia Appendix 4 displays the summary of the results regarding glycemic control outcomes.

Glycated Hemoglobin $\mathrm{A}_{1 \mathrm{c}}\left(\mathrm{HbA}_{1 \mathrm{c}}\right)$ ( $n=164$ intervention group (IG), $n=161$ control group (CG)). Overall, the intervention patients showed better $\mathrm{HbA}_{1 \mathrm{c}}$ values than the control groups $[23,24]$. Guo et al. displayed a significant difference in favor of the intervention group $(-1.3 \%$ intervention group versus $-0.6 \%$ control group, $P<.001$ ) [23], .whilst Mackillop et al. recognized a slight increase in both groups $(0.02 \%$ per 28 days in the intervention group verses $0.03 \%$ per 28 days in the control group, $95 \%$ CI -0.05 to 0.03$)$ [24].

Fasting blood glucose (FBG) ( $n=69$ IG, $n=62$ CG). Significant improvements of FBG were found by Yang et al. $(P<.001)$ and Bromuri et al. $(P<.001)$ favoring the intervention group [22, 25].

1-h Postprandial blood glucose (PBG) $(n=57$ IG, $n=50$ CG). Yang et al. displayed lower, but not significantly, PBG values in the intervention compared to control group $(7.71 \pm 0.73 \mathrm{mmol} / \mathrm{L} \mathrm{IG}$ verses $7.75 \pm 2.08 \mathrm{mmol} / \mathrm{L}$ CG, $P=.780)[25]$.

2-h Postprandial blood glucose (PBG) $(n=69$ IG, $n=62$ CG). There were significant improvements of $2 \mathrm{~h}-\mathrm{PBG}$ values after the interventions [22, 25]. Yang et al. showed a significant difference in favor of the intervention group $(P<.001)$ [25]. Bromuri et al. reported significant differences for morning $(P<.001)$ and noon $(P<.001)$ favoring the intervention group [22].

2-h Oral Glucose Tolerance Test (OGTT) $(n=176$ IG, $n=181$ CG). Neither Guo $(P=.638)$ et al. nor Borgen et al. $(P=.22)$ found significant differences between groups [21, 23].

Mean blood glucose ( $n=170$ IG, $n=157$ CG). There were significant improvements regarding mean blood glucose [Bromuri et al. $(P<.001)$ and Miremberg et al. $(P<.001)][16,22]$. However, Mackillop et al. found improved blood glucose values, but no significant differences between groups regarding the rate of change of blood glucose $(-0.16 \mathrm{mmol} / \mathrm{L}$ in intervention group versus $-0.14 \mathrm{mmol} / \mathrm{L}$ in control group, $P=.78$ ) [24].

Patient compliance (ratio between actual and instructed blood glucose measurements $\times 100)(n=124$ IG, $n=120$ CG). Significant improvements were shown in both studies [Guo et al. $(P<.001)$ and Miremberg et al. $(P<.001)][16,23]$.

Off-target FBG measurements $(\mathrm{n}=124$ IG, $\mathrm{n}=120$ CG). Miremberg et al. $(P<.001)$ as well as Guo et al. $(P<.001)$ displayed significant differences in favor of the intervention group [16, 23].

Off-target 1 h-PBG measurements $(n=60$ IG, $n=60$ $C G)$. Miremberg et al. found significant differences in favor of the intervention group $(P<.001)$ [16].

Off-target $2 \mathrm{~h}$-PBG measurements $(n=64$ IG, $\mathrm{n}=60$ CG). displaying significant differences favoring the intervention group $(P<.001)$ [23].

\section{Pregnancy- and birth-related outcomes}

The findings of pregnancy and birth related outcomes are displayed in Multimedia Appendix 5.

Pregnancy-induced hypertension and/or preeclampsia $(n=218$ IG, $n=212$ CG). Although the intervention groups showed lower rates regarding the outcome, no significant differences were found by Mackillop et al. $(P=.22)$, Miremberg et al. $(P>.99)$ or Yang et al. $(P=.347)$.

Preterm birth ( $n=158$ IG, $n=152$ CG). There were fewer preterm births in the intervention groups, but neither Yang et al. $(P=.248)$ nor Mackillop et al. $(P>.05)$ did find significant differences.

Induction of labor ( $n=172$ IG, $n=181 \mathrm{CG})$. Though one study reported fewer incidents of induction of labor in the intervention group [21], no significant differences were found by Borgen et al. $(P=.33)$ or Miremberg et al. $(P=.248)[16,21]$.

Shoulder dystocia ( $n=220$ IG, $n=222$ CG). In all three studies only one incident of shoulder dystocia occurred $[16,23,24]$.

Mode of delivery ( $n=390$ IG, $n=393$ CG). Borgen et al. displayed significant differences $(P=.03)$ and found obviously fewer operative vaginal deliveries and emergency caesarean sections, and more spontaneous vaginal deliveries and planned caesarean sections in the intervention group [21]. Mackillop et al. reported significant differences $(P=.005)$ as well and recognized more vaginal deliveries and a lower rate of caesarean sections, especially of emergency caesarean sections, in the intervention group [24]. Guo et al., Miremberg et al. and Yang et al. did not find any significant differences [16, 23, 25]. 


\section{Neonatal outcomes}

The results regarding these outcomes are shown in Multimedia Appendix 6.

Birth weight. The results showed a trend towards lower birth weight in the intervention groups, but no significant differences were found by Borgen et al. $(P=.69)$, Mackillop et al. $(P=.18)$, Miremberg et al. $(P=.878)$ or Yang et al. $(P=.988)[16,21,24,25]$.

Macrosomia. Fewer incidents of macrosomia occurred in the intervention groups compared with the control groups, but no significant differences were found by Guo et al. $(P=.295)$ or Yang et al. $(P=.542)[23,25]$.

Large for gestational age (LGA). Mackillop et al. did not report the concrete number of incidents and did not find significant differences $(P>.05)$, whereas Miremberg et al. reported the same amount of LGA in the intervention and control group $(P>.99)[16,24]$.

Hypoglycemia ( $n=277$ IG, $n=263 \mathrm{CG})$. Fewer incidents of hypoglycemia of the newborn were reported favoring the intervention groups but not significant [23, 25]. Yang et al. did not report on significance in this outcome [25]. No significant differences were found by Guo et al. $(P=.185)$, Mackillop et al. $(P=.42)$ or Miremberg et al. $(P>.99)[16,23,24]$.

Admission to higher level of care $(n=330$ IG, $\mathrm{n}=330$ CG). The studies showed a trend towards being fewer often transferred to neonatal intensive care units favoring the intervention group. Neither Borgen et al. $(P=.38)$, Mackillop et al. $(P=.08)$, Miremberg et al. $(P>.99)$ nor Yang et al. $(P=.657)$ found significant differences between groups [16, 21, 24, 25].

\section{Discussion}

In total, especially glycemic control and pregnancy and birth related outcomes showed improving trends through using GDM specific mHealth-apps. In detail, compared to control groups, fasting blood glucose, 2-h postprandial blood glucose, off target blood glucose measurements, delivery mode (more vaginal deliveries and fewer (emergency) caesarean sections) and patient compliance improved by using mHealth-apps in GDM care. We found only a few RCTs and CCTs evaluating the clinical effectiveness of GDM specific mHealth-Apps. Despite the limited data, the included studies indicated that GDM specific mHealth-Apps show a trend towards improving the management of GDM.

Especially more data in terms of parameters that stick out the most in terms of diabetic but also pregnancy/maternity as well as neonatal health $\left(\mathrm{eg}, \mathrm{HbA}_{1 \mathrm{c}}\right.$, FBG, PBG, OGTT, oral glucose challenge test (OGCT) [26]) are needed. However, in our review each of those parameters was only investigated in one or two studies, except for the OGCT. A standardized set of investigated outcomes is needed to extend the evidence on the effectiveness of GDM specific mHealth-Apps. In addition to that, nutritional and physical activity outcomes should be anlayzed as well, since these are basic treatments in terms of the management and control of GDM [27-29].

We assume that mHealth-apps enhance the compliance, empower the patients, and enable a more intensive and closely monitored therapy.

\section{Glycemic control parameters}

Overall, the results showed an obvious trend in improving glycemic control parameters. Both studies, that evaluated $\mathrm{HbA}_{1 \mathrm{c}}$, displayed lower $\mathrm{HbA}_{1 \mathrm{c}}$ values respectively a lower rate of change in the intervention group [23, 24]. FBG results show an improvement through GDM specific mHealth-Apps, both studies reporting significant differences between the groups [22, 25]. But the quality of both studies was rated as weak. Regarding the PBG, findings on 1-h after glucose or nutrition administration are lacking. None of the studies reported on the OGTT values $60 \mathrm{~min}$ after glucose administration and only one study included the 1h-PBG [25]. But Bromuri et al. and Yang et al. found significant $2 \mathrm{~h}-\mathrm{PBG}$ improvements in their intervention groups [22, 25]. Though, the results are restricted by the fact that quality of both studies was rated as weak. Moreover, an effect on OGTT value 120 min after glucose administration needs further investigation. Improvements were displayed in the intervention and control groups and none of the studies found significant differences between groups [21, 23]. Furthermore, the results displayed a clear effect of GDM specific mHealth-Apps on mean blood glucose values, since all studies found improvements in their intervention groups $[16,22,24]$. In addition, an improving trend of patient compliance could be observed, since Guo et al. and Miremberg et al. found significant improvements and got a study quality rating as moderate $[16,23]$. Moreover, there is a positive trend in reducing off-target measurements in terms of FBG, 1 h-PBG and $2 \mathrm{~h}$-PBG $[16,23]$.

\section{Pregnancy- and birth-related outcomes}

Mode of delivery was the only pregnancy and birthrelated outcome in which significant differences were found [21, 24]. However, against this background the use of a GDM specific mHealth-App may improve GDM management and leads to fewer emergency caesarean sections and more vaginal deliveries [21,24].

Moreover, a positive effect on induction of labor, preterm birth, pregnancy-induced hypertension and/or preeclampsia might be possible. Although none of the studies reported significant differences regarding one of these outcomes, most of them showed improved rates 
of incidents in the intervention compared with control groups [16, 21, 23-25]. No trend regarding shoulder dystocia can be derived, since the outcome occurred only once in one study, even though three studies included shoulder dystocia as an outcome [16, 23, 24].

\section{Neonatal outcomes}

The use of GDM specific mHealth-Apps may improve birth weight or incidence of macrosomia. The studies found no significant differences [16, 21, 23-25]. But some studies showed a promising trend towards lower birth weight or fewer incidents of macrosomia in the intervention groups $[16,21,23,25]$.

In addition to that and more importantly, the results display a trend towards infants being fewer often transferred to neonatal intensive care units by using mHealthapps in GDM care [16, 21, 24, 25]. No clear trend regarding hypoglycemia of the newborn can be derived [23-25].

\section{Comparison with prior work}

Skar et al. found that a GDM specific mHealth-App can increase the confidence of women with GDM in their self-management and improve their motivation for behavioral changes [30]. Moreover, Chen et al. conclude in their scoping review that GDM specific mHealth-Apps can provide time- and cost-efficient personalized interventions to improve GDM management [31]. Although it is not shown in the included studies, an improved GDM management can reduce the risks of LGA, shoulder dystocia or hypertensive disorders [32, 33]. In addition, Xie et al. [17] noted that telemedicine interventions (including app-based interventions) can decrease the glycemic levels of GDM patients effectively and also reduce the risk of maternal, fetal and neonatal complications.

Other studies that did not specifically examine apps, but rather telemedical web and internet-based interventions as part of GDM management, showed promising trends.

For example, Rasekaba et al. [34] concluded that glycemic control indicated an improving trend in favor of telemedicine. The authors indicated advantages of telemetric systems in the reduction of face-to-face and unscheduled consultations. Moreover, Ming et al. [35] analyzed telemedicine technologies for diabetes in pregnancy showing a significant $\mathrm{HbA}_{1 \mathrm{c}}$ reduction of $-1.14 \%$ (mean difference) ( $95 \%$ CI: 0.25 to 0.04 ).

\section{Limitations}

There are some limitations that should be noted: the available literature is very limited and further limited by discrepancies between investigated outcomes. The included studies were published relatively recently, the oldest being from 2016. This suggest that the topic has become more and more important over the past years. Because of that, we expect more publications soon. Heterogeneity in the investigated outcomes show that a more standardized set of outcomes, especially regarding glycemic control parameters is needed. In addition to that, the quality of the included papers is moderate to weak. There are only a few studies in the area of GDM and apps; due to the small number, the results should be viewed with caution.

\section{Conclusions}

Since pregnant women tend to use and accept mHealthApps as part of accompanying pregnancy, further research on the full potential of GDM specific mHealthApps is needed [14, 15]. mHealth-Apps improved the management of other types of diabetes mellitus as well $[36,37]$.

In total, especially glycemic control outcomes showed improving trends through using GDM specific mHealthapps. Fasting blood glucose, 2-h postprandial blood glucose, off target blood glucose measurements, delivery mode (more vaginal deliveries and fewer (emergency) caesarean sections) and patient compliance enhanced by using mHealth-apps in GDM management. Despite the limited data, mHealth-Apps showed potential to improve the management of GDM.

\section{Abbreviations}

CCT: Controlled clinical trial; CG: Control group; EPHPP: Effective Public Health Practice Project; FBG: Fasting blood glucose; GDM: Gestational diabetes mellitus; $\mathrm{HbA}_{1}$ : Glycated hemoglobin $\mathrm{A}_{1}$; IG: Intervention group; MA: Metaanalysis; $m$ Health: Mobile Health; MQ: Moderate quality; PRISMA: Preferred Reporting Items for Systematic Reviews and Meta-Analyses; RCT: Randomized controlled trial; T2D: Type 2 diabetes mellitus; WQ: Weak quality.

\section{Supplementary Information}

The online version contains supplementary material available at https://doi. org/10.1186/s12884-021-04274-7.

Additional file 1: Figure 1: PRISMA flowchart. Table 1: Summary of included studies. Table 2: Appraisal of included studies. Table 3: Glycemic control outcomes. Table 4: Pregnancy and birth outcomes. Table 5: Neonatal outcomes.

\section{Acknowledgments}

This manuscript was created in the context of the project with the number EB 440/4-1 by the German Research Foundation (Deutsche Forschungsgemeinschaft, DFG). Therefore, we would like to thank the DFG for the strong support of this research work.

\section{Authors' contributions}

C.E. designed the systematic review. C.E., M.L. and S.S. performed the systematic literature search and data extraction of all included studies and wrote the manuscript. C.E. provided guidance in synthesizing and interpreting the study results. C.E. reviewed the manuscript for intellectual content and approved the final manuscript. 


\section{Funding}

German Research Foundation (Deutsche Forschungsgemeinschaft, DFG), project number EB 440/4-1. Open Access funding enabled and organized by Projekt DEAL.

\section{Availability of data and materials}

All data generated and analyzed during are included within the article and its supplementary information files.

\section{Declarations}

Ethics approval and consent to participate

Not applicable.

\section{Consent for publication}

Not applicable.

\section{Competing interests}

The authors declare that they have no competing interests.

Received: 18 May 2021 Accepted: 15 November 2021

Published: 5 December 2021

\section{References}

1. International Diabetes Federation. Gestational diabetes; 2020. https:// www.idf.org/our-activities/care-prevention/gdm. Accessed 8 Jun 2020

2. International Diabetes Federation. Prevalence of gestational diabetes mellitus (GDM); 2019. https://diabetesatlas.org/data/en/indicators/14/. Accessed 8 Jun 2020

3. Ferrara A. Increasing prevalence of gestational diabetes mellitus: a public health perspective. Diabetes Care. 2007;30(Suppl 2):S141-6. https://doi. org/10.2337/dc07-s206.

4. Zhu Y, Zhang C. Prevalence of gestational diabetes and risk of progression to type 2 diabetes: a global perspective. Curr Diab Rep. 2016;16:7. https:// doi.org/10.1007/s11892-015-0699-x.

5. Chiefari E, Arcidiacono B, Foti D, Brunetti A. Gestational diabetes mellitus: an updated overview. J Endocrinol Investig. 2017;40:899-909. https://doi. org/10.1007/s40618-016-0607-5.

6. Metzger BE. Long-term outcomes in mothers diagnosed with gestational diabetes mellitus and their offspring. Clin Obstet Gynecol. 2007;50:972-9. https://doi.org/10.1097/GRF.0b013e31815a61d6.

7. West NA, Crume TL, Maligie MA, Dabelea D. Cardiovascular risk factors in children exposed to maternal diabetes in utero. Diabetologia. 2011;54:504-7. https://doi.org/10.1007/s00125-010-2008-1.

8. Tam WH, Ma RCW, Yang X, Ko GTC, Tong PCY, Cockram CS, et al. Glucose intolerance and cardiometabolic risk in children exposed to maternal gestational diabetes mellitus in utero. Pediatrics. 2008;122:1229-34. https://doi.org/10.1542/peds.2008-0158.

9. Eberle C. Fetale Programmierung des diabetes mellitus Typ 2 : intrauterine Wachstumsretardierung als Risikofaktor? MMW Fortschr Med. 2010;152:76-82. https://doi.org/10.1007/BF03373173.

10. Eberle C, Ament C. Diabetic and metabolic programming: mechanisms altering the intrauterine milieu. ISRN Pediatr. 2012;2012:975685. https:// doi.org/10.5402/2012/975685.

11. Stupin J, Schäfer-Graf U, Hummel M. Diabetes in der Schwangerschaft: Praxisorientiertes Wissen zu Gestationsdiabetes, diabetes mellitus Typ 1 und 2, MODY. 1st ed; 2020.

12. Meister S, Becker S, Leppert F, Drop L. Digital health, Mobile health und co. - Wertschöpfung durch Digitalisierung und Datenverarbeitung. In: Pfannstiel MA, Da-Cruz P, Mehlich H, editors. Digitale transformation von Dienstleistungen im Gesundheitswesen I: impulse für die Versorgung. Wiesbaden: Springer Gabler; 2017. p. 185-212. https://doi.org/10.1007/ 978-3-658-12258-4_13.

13. Eberle C, Ament C. Individualisierte Nutzung von diabetes-spezifischen mHealth-apps. Diabetologie und Stoffwechsel. 2015. https://doi.org/10. 1055/s-0035-1549653.

14. Eberle C, Ament C. Schwangerschaft und Digitalisierung - Individualisierte Nutzungsanalyse von Schwangerschafts- und GDM-apps.
In: Diabetes Kongress 2019-54. Jahrestagung der DDG; 5/29/20196/1/2019. Berlin: Georg Thieme Verlag KG; 2019. https://doi.org/10. 1055/s-0039-1688270.

15. Eberle C, Ament C. Diabetes \& Schwangerschaft 4.0 - Individualisierte Nutzungsanalyse von mHealth-apps. Diabetologie und Stoffwechsel. 2017. https://doi.org/10.1055/s-0037-1601701.

16. Miremberg H, Ben-Ari T, Betzer T, Raphaeli H, Gasnier R, Barda G, et al. The impact of a daily smartphone-based feedback system among women with gestational diabetes on compliance, glycemic control, satisfaction, and pregnancy outcome: a randomized controlled trial. AJOG. 2018;218:453.e1-7. https://doi.org/10.1016/j.ajog.2018.01.044.

17. Xie W, Dai P, Qin Y, Wu M, Yang B, Yu X. Effectiveness of telemedicine for pregnant women with gestational diabetes mellitus: an updated meta-analysis of 32 randomized controlled trials with trial sequential analysis. BMC Pregnancy Childbirth. 2020;20:198. https:/doi.org/10.1186/ s12884-020-02892-1.

18. Moher D, Liberati A, Tetzlaff J, Altman DG. Preferred reporting items for systematic reviews and meta-analyses: the PRISMA statement. PLoS Med. 2009;6:e1000097. https://doi.org/10.1371/journal.pmed.1000097.

19. Ciliska D, Micucci S, Dobbins M, Thomas BH. Quality assessment tool for quantitative studies; 2010.

20. Thomas BH, Ciliska D, Dobbins M, Micucci S. A process for systematically reviewing the literature: providing the research evidence for public health nursing interventions. Worldviews Evid-Based Nurs. 2004;1:176-84. https://doi.org/10.1111/j.1524-475X.2004.04006.x.

21. Borgen I, Småstuen MC, Jacobsen AF, Garnweidner-Holme LM, Fayyad S, Noll J, et al. Effect of the pregnant+ smartphone application in women with gestational diabetes mellitus: a randomised controlled trial in Norway. BMJ Open. 2019;9:e030884. https://doi.org/10.1136/bmjop en-2019-030884.

22. Bromuri S, Puricel S, Schumann R, Krampf J, Ruiz J, Schumacher M. An expert personal health system to monitor patients affected by gestational diabetes mellitus: a feasibility study. AIS. 2016;8:219-37. https://doi. org/10.3233/AIS-160365.

23. Guo H, Zhang Y, Li P, Zhou P, Chen L-M, Li S-Y. Evaluating the effects of mobile health intervention on weight management, glycemic control and pregnancy outcomes in patients with gestational diabetes mellitus. J Endocrinol Investig. 2019;42:709-14. https://doi.org/10.1007/ s40618-018-0975-0.

24. Mackillop L, Hirst JE, Bartlett KJ, Birks JS, Clifton L, Farmer AJ, et al. Comparing the efficacy of a Mobile phone-based blood glucose management system with standard Clinic Care in Women with gestational diabetes: randomized controlled trial. JMIR Mhealth Uhealth. 2018;6:e71. https://doi.org/10.2196/mhealth.9512.

25. Yang P, Lo W, He Z-L, Xiao X-M. Medical nutrition treatment of women with gestational diabetes mellitus by a telemedicine system based on smartphones. J Obstet Gynaecol Res. 2018;44:1228-34. https://doi.org/ 10.1111/jog.13669.

26. Donovan L, Hartling L, Muise M, Guthrie A, Vandermeer B, Dryden DM. Screening tests for gestational diabetes: a systematic review for the U.S. preventive services task force. Ann Intern Med. 2013;159:115-22. https:// doi.org/10.7326/0003-4819-159-2-201307160-00657.

27. Padayachee C, Coombes JS. Exercise guidelines for gestational diabetes mellitus. World J Diabetes. 2015;6:1033-44. https://doi.org/10.4239/wjd. v6.i8.1033.

28. Hu Z-G, Tan R-S, Di Jin LW, Zhou X-Y. A low glycemic index staple diet reduces postprandial glucose values in Asian women with gestational diabetes mellitus. JIM. 2014;62:975-9. https://doi.org/10.1097/JIM.00000 00000000108.

29. Viana LV, Gross JL, Azevedo MJ. Dietary intervention in patients with gestational diabetes mellitus: a systematic review and meta-analysis of randomized clinical trials on maternal and newborn outcomes. Diabetes Care. 2014;37:3345-55. https://doi.org/10.2337/dc14-1530.

30. Skar JB, Garnweidner-Holme LM, Lukasse M, Terragni L. Women's experiences with using a smartphone app (the pregnant+ app) to manage gestational diabetes mellitus in a randomised controlled trial. Midwifery. 2018;58:102-8. https://doi.org/10.1016/j.midw.2017.12.021.

31. Chen Q, Carbone ET. Functionality, implementation, impact, and the role of health literacy in Mobile phone apps for gestational diabetes: scoping review. JMIR Diabetes. 2017;2:e25. https://doi.org/10.2196/diabetes.8045. 
32. Landon MB, Spong CY, Thom E, Carpenter MW, Ramin SM, Casey B, et al. A multicenter, randomized trial of treatment for mild gestational diabetes. N Engl J Med. 2009;361:1339-48. https://doi.org/10.1056/NEJMoa0902 430.

33. Crowther CA, Hiller JE, Moss JR, McPhee AJ, Jeffries WS, Robinson JS. Effect of treatment of gestational diabetes mellitus on pregnancy outcomes. N Engl J Med. 2005;352:2477-86. https://doi.org/10.1056/NEJMo a042973.

34. Rasekaba TM, Furler J, Blackberry I, Tacey M, Gray K, Lim K. Telemedicine interventions for gestational diabetes mellitus: a systematic review and meta-analysis. Diabetes Res Clin Pract. 2015;110:1-9. https://doi.org/10. 1016/j.diabres.2015.07.007.

35. Ming W-K, Mackillop LH, Farmer AJ, Loerup L, Bartlett K, Levy JC, et al. Telemedicine Technologies for Diabetes in pregnancy: a systematic review and meta-analysis. J Med Internet Res. 2016;18:e290. https://doi.org/10. 2196/jmir.6556.

36. Fu H, McMahon SK, Gross CR, Adam TJ, Wyman JF. Usability and clinical efficacy of diabetes mobile applications for adults with type 2 diabetes: a systematic review. Diabetes Res Clin Pract. 2017;131:70-81. https://doi. org/10.1016/j.diabres.2017.06.016

37. Cui M, Wu X, Mao J, Wang X, Nie M. T2DM self-management via smartphone applications: a systematic review and meta-analysis. PLoS One. 2016;11:e0166718. https://doi.org/10.1371/journal.pone.0166718.

\section{Publisher's Note}

Springer Nature remains neutral with regard to jurisdictional claims in published maps and institutional affiliations.

- fast, convenient online submission

- thorough peer review by experienced researchers in your field

- rapid publication on acceptance

- support for research data, including large and complex data types

- gold Open Access which fosters wider collaboration and increased citations

- maximum visibility for your research: over $100 \mathrm{M}$ website views per year

At BMC, research is always in progress.

Learn more biomedcentral.com/submissions 